ARTICLE

https://doi.org/10.1038/s41467-020-17321-2

\title{
Structurally divergent dynamic combinatorial chemistry on racemic mixtures
}

\author{
Tiberiu-M. Gianga (1) ${ }^{1,2} \&$ G. Dan Pantoș (i) ${ }^{1 凶}$
}

Structurally Divergent Reactions on Racemic Mixtures are atypical processes in Nature. The few examples reported in the literature take place in organic solvents and are driven by the reagents' interaction with bulky chiral catalysts. Herein, we describe a dynamic combinatorial approach to generate structural divergence from racemic building blocks. The divergence is due to a stereospecific electron-donor - electron-acceptor interaction of diastereomeric macrocycles, leading to structurally distinct pseudorotaxanes. The equilibrated dynamic combinatorial library contains, amongst various macrocycles, two different types of [2] catenanes that are non-isomeric. The formation of these [2]catenanes is due to a spontaneous stereo and structurally divergent assembly of the building blocks.

\footnotetext{
${ }^{1}$ Department of Chemistry, University of Bath, Bath BA2 7AY, UK. ${ }^{2}$ Present address: Beamline B23, Diamond Light Source, Ltd., Chilton, Didcot OX11 ODE, UK. ${ }^{凶}$ email: g.d.pantos@bath.ac.uk
} 
S tructurally divergent reactions on racemic mixtures (SDRRMs) utilise an enantiomeric mixture to produce two distinct chemical entities. This type of reaction is contrasting the predictable formation of diastereomers when two chiral entities react. SDRRM produces chemical species that are either structural isomers or non-isomeric. These species have different enthalpies of formation from starting materials that are isoenergetic. The energetic divergence observed in SDRRMs is due to each enantiomer following a different reaction pathway, and thus allowing for the formation of structurally different products. The energetic and structural divergence that occurs when these racemic mixtures react with a chiral molecule can provide insight into Nature's chiral propagation question, as it explains how a small change at the molecular level leads to vastly different products.

To the best of our knowledge, only four SDRRMs ${ }^{1}$ have been reported in the literature ${ }^{2-8}$; of these, just two ${ }^{3,4}$ lead to nonisomeric structures. The structural divergence was usually achieved through the ability of one of the diastereomeric products to undergo a rearrangement or participate in a subsequent reaction with another compound present in the reaction mixture. The four known SDRRMs were developed in organic solvents and relied on structural constraints imposed by a bulky chiral catalyst.

We describe herein an aqueous SDRRM in a dynamic combinatorial environment to generate structural divergence from racemic building blocks. The reactions take place under the conditions of disulphide exchange in aqueous dynamic combinatorial libraries (DCL) and the resulting products are in stark contrast to our previous work, where enantiomers lead to the formation of diastereomers when reacted with a chiral building block $^{9,10}$. In this dynamic combinatorial chemistry (DCC) system, the divergence is due to a combination of the assembly of diastereomeric macrocycles and a stereospecific electron-donor $(D)$ - electron-acceptor $(A)$ interaction, leading to structurally distinct pseudorotaxanes. These factors, combined with a difference in the stability of the final products, lead to the spontaneous assembly of two structurally different, non-isomeric [2]catenanes: one homochiral with a $D A A D$ aromatic stack (Cat I RRRR), while the other is heterochiral with a $D A D D$ stack (Cat II RSRR). This work enhances our understanding of SDRRMs and raises the possibility of supramolecular interactions in aqueous media, playing a crucial role in biological homochirality ${ }^{11-13}$.

\section{Results}

Building block design. The building blocks used throughout this study have cysteine appendages for disulphide exchange and water solubility, having either a naphthalenediimide (NDI, electron-acceptor) or benzodithiophene (BDT, electron-donor) cores. The enantiomeric NDIs $(R, R-\mathbf{1}, S, S-\mathbf{1})$ are readily available ${ }^{14}$, and have extensively been used in dynamic combinatorial chemistry $9,15-19$. The seven step synthesis of BDT, $R, R-2$, is reported here (see Supplementary Fig. 1). The BDT core is an electron-donor ${ }^{20,21}$ with a large negative quadrupole moment $(-23 \mathrm{~B})$, thus making it an ideal partner for aromatic stacking interactions with the electron-acceptor NDI, $Q_{\mathrm{zz}}=14.2 \mathrm{~B}$. A key feature in the structure of BDT is the presence of the two methoxy groups in positions 3 and 7 , which form strong intramolecular $\mathrm{H}$-bonds with the amide $\mathrm{NH}$ groups, thus further rigidifying the structure and making it a quasi-pentacyclic fused system. The additional rigidity makes the BDT building block more susceptible to the hydrophobic effect. The DCLs were set up by dissolving the building blocks in $10 \mathrm{mM}$ aqueous $\mathrm{NaOH}$ to a total concentration of $5 \mathrm{mM}$. The $\mathrm{pH}$ was adjusted to 8 using a stock solution of $100 \mathrm{mM} \mathrm{NaOH} / 100 \mathrm{mM} \mathrm{HCl}$. The DCLs were stirred in the presence or absence of salt additives, and/or competitors (vide infra) for three days under air in capped vials to allow full oxidation of the building blocks. The DCL composed of $1.25 \mathrm{mM} R, R-\mathbf{1}, 1.25 \mathrm{mM} \mathrm{S,S-1}$ and $2.5 \mathrm{mM} R, R-\mathbf{2}$ contained, after the equilibrium was reached, a complex mixture of macrocycles and [2]catenanes. A detailed analysis revealed that the two enantiomers of $\mathbf{1}$ predominantly formed non-isomeric species. One of the most striking differences is the formation of the $D A A D$ [2] catenane (Cat I RRRR), containing two NDI $(R, R-\mathbf{1})$ and two BDT cores, while the $S, S-1$ building block led to the formation of the $D A D D$ [2]catenane (Cat II RSRR), which contains one NDI $(S, S-\mathbf{1})$ and three BDT cores (Fig. 1). These [2] catenanes are non isomeric despite one common (BDT) building block interacting with enantiomeric species (NDIs $R, R-\mathbf{1}$ and $S, S-1)$. While the synthesis of [2] catenanes is in and of itself exciting, this work is set apart by the discovery of a different type of SDRRM driven by supramolecular interactions. This can open the door for the development of other divergent reactions on racemic mixtures based on aqueous, metal-free chemistry.

Homochiral DCL. To further interrogate this outcome, we set up DCLs containing $R, R-2$ and each of the NDI enantiomers separately. The $R, R-1$ (NDI) and $R, R-2$ (BDT) library led, in the presence of $1 \mathrm{M} \mathrm{NaNO}_{3}$, to the assembly of the DAAD [2]catenane (Cat I RRRR) in $76 \%$ of the total library distribution (Fig. 2, for full characterisation see Supplementary Figs. 26-35). Sodium nitrate was added in order to increase the hydrophobic effect which leads to an increase in the yield of interlocked species (Supplementary Fig. 26) 16,18,22

Heterochiral DCL. Contrasting the behaviour described above, the $1: 1 S, S-1: R, R-2$ library led, in the presence of $1 \mathrm{M} \mathrm{NaNO}_{3}$, to the assembly of the DADD [2] catenane (Cat II RSRR) in $21 \%$ of the total library distribution (Fig. 3, for full characterisation see Supplementary Figs. 36-46). Changing the building block stoichiometry to $1: 3 S, S-1: R, R-2$, increased the proportion of Cat II RSRR up to $41 \%$. The other major library members consisted of a donor dimer (Y), DA heterodimer (Z) and a DAAA (RSSS) macrocycle based on three $S, S-1$ and one $R, R-2$ molecules.

Once we characterised the outcomes of the racemic (Fig. 1) and enantiopure DCLs (Figs. 2, 3), we investigated in detail the behaviour of this interesting set of libraries along with other DCLs in which we varied the ratio of the donor and acceptor building blocks, the solvent polarity and composition (Supplementary Figs. 71, 74, 81). The DCL of a racemic mixture of 1 and $R, R-2$ is an SDRRM because, with the exception of the DAAD catenane and the two $D A$ heterodimers where we see some erosion of chirality, all of the other library members contain either only one of the enantiomers of 1 or a mixture of $R, R-\mathbf{1}, S, S$ 1 and $R, R-2$. All libraries have been studied at regular intervals until equilibration/full oxidation has been achieved (Supplementary Figs. 78-80). This allowed us to monitor and understand the evolution of all species involved in the assembly of Cat I RRRR and Cat II RSRR. Figure 4 depicts the major species and equilibria involved in their assembly (a full depiction of the possible library members-up to tetramers-is presented in Supplementary Fig. 91) 23,24

The kinetic study of the DCL evolution of $R, R-1$ and $R, R-2$ gives insight into the mechanism leading to the efficient assembly of Cat I RRRR (Supplementary Figs. 56-57). As expected, the first species formed in the DCL is the linear heterodimer, which is closely followed by the cyclic heterodimer (X, Fig. 2). Once a critical concentration of these two species is reached, Cat I RRRR starts to form, presumably by the threading of the linear heterodimer through the cyclic analogue, and subsequent final oxidative ring closing. 
a
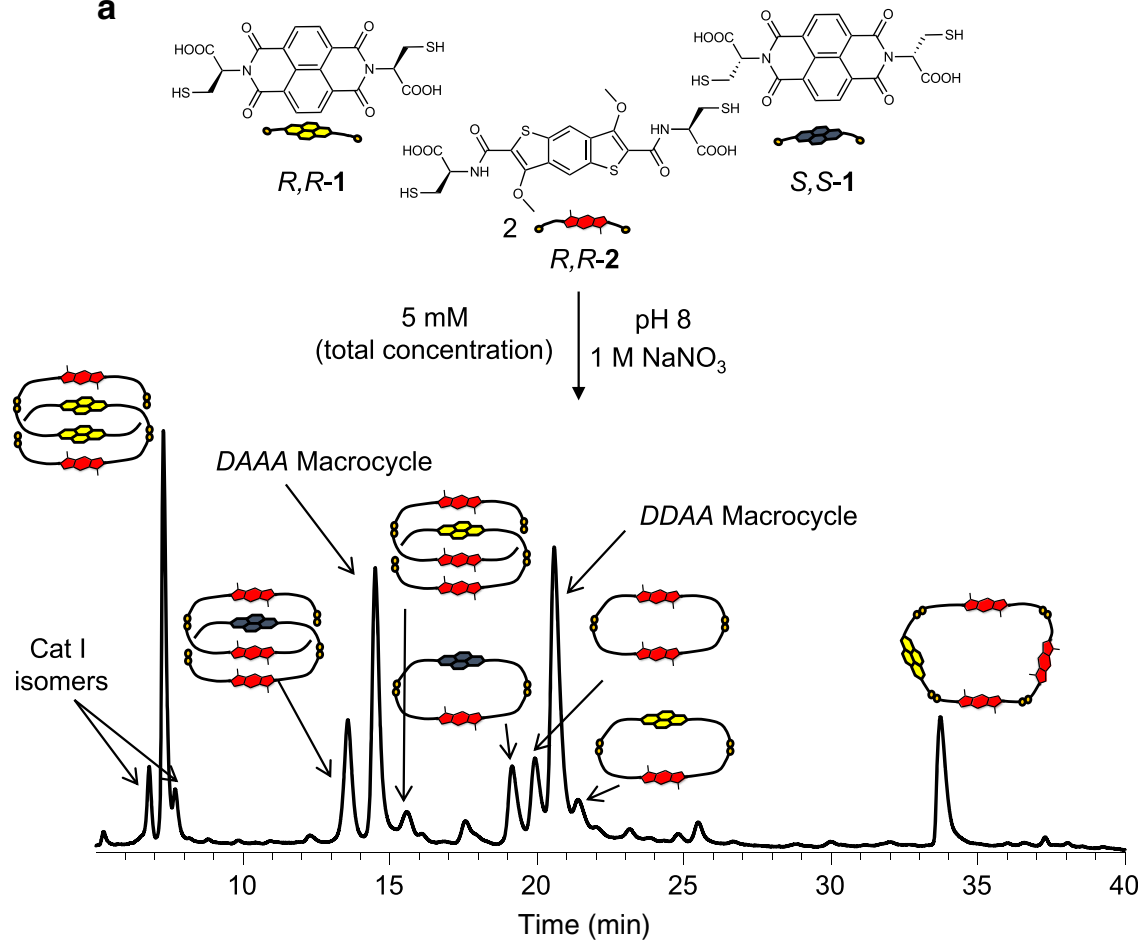

b

\begin{tabular}{|c|c|c|c|c|c|}
\hline Species & & 00 & & & \\
\hline i.d. & $\begin{array}{l}\text { Cat I } \\
\text { RRRR }\end{array}$ & $\begin{array}{c}\text { Cat I } \\
\text { RRSR }\end{array}$ & $\begin{array}{l}\text { Cat I } \\
\text { RSSR }\end{array}$ & $\begin{array}{l}\text { Cat II } \\
\text { RSRR }\end{array}$ & $\begin{array}{l}\text { Cat II } \\
\text { RRRR }\end{array}$ \\
\hline$\%$ in $\mathrm{DCL}$ & $\begin{array}{l}13.67 \\
(0.03)\end{array}$ & \multicolumn{2}{|c|}{$\begin{array}{c}2.52(0.03)+2.16 \\
(0.07)\end{array}$} & $7.68(0.09)$ & $2.33(0.05)$ \\
\hline d.r. & \multicolumn{3}{|c|}{$75: 14: 11$} & \multicolumn{2}{|c|}{$77: 23$} \\
\hline \multicolumn{6}{|c|}{ Total proportion of [2]catenane: $28.36 \%$} \\
\hline
\end{tabular}

C

\begin{tabular}{|c|c|c|c|c|}
\hline Species & & & \\
\hline i.d. & $\mathbf{X}$ & $\mathbf{Y}$ & $\mathbf{Z}$ & NDI Dimer \\
\hline$\%$ in DCL & $7.54(0.18)$ & $8.53(0.13)$ & $5.63(0.14)$ & $2.82(0.07)$ \\
\hline \multicolumn{5}{|c|}{ Total proportion of dimers: $24.52 \%$} \\
\hline
\end{tabular}

d

\begin{tabular}{|c|c|c|c|c|c|}
\hline Species & $\begin{array}{c}D A A A \mathrm{I} \\
\text { Macrocycle }\end{array}$ & $\begin{array}{c}D A A A \text { II } \\
\text { Macrocycle }\end{array}$ & $\begin{array}{c}D D A A \\
\text { Macrocycle }\end{array}$ & & \\
\hline$\%$ in $\mathrm{DCL}$ & $\begin{array}{l}15.92 \\
(0.14)\end{array}$ & $1.77(0.01)$ & $18.46(0.17)$ & $\begin{array}{c}1.62 \\
(0.03)\end{array}$ & $\begin{array}{c}9.35 \\
(0.04)\end{array}$ \\
\hline
\end{tabular}

Fig. 1 Racemic DCL. a Reverse-phase HPLC analysis at $389 \mathrm{~nm}$ of $R, R-\mathbf{1}: S, S-\mathbf{1}: R, R-\mathbf{2}$ (1:1:2 molar ratio, $5 \mathrm{mM}$ total concentration) library in the presence of 1 $\mathrm{M} \mathrm{NaNO}_{3}$; $\mathbf{b}$ the table shows the [2]catenanes i.d., percentages, diastereomeric ratios, and the total proportions of [2]catenanes in the DCL; $\mathbf{c}$ the table shows the dimers i.d., percentages and the total proportions of [2]catenanes in the DCL; $\mathbf{d}$ the table shows the macrocycles i.d., percentages and the total proportions of [2]catenanes in the DCL. The integrations were done in triplicate and the RMSD is reported in parenthesis along with the value. For a complete description of the library, see Supplementary Fig. 71. 
a

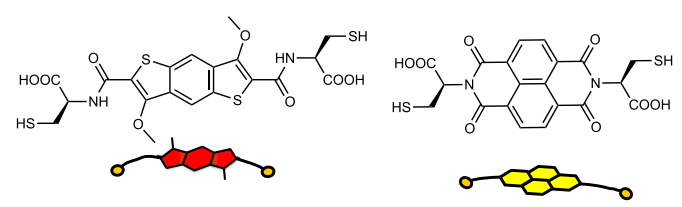

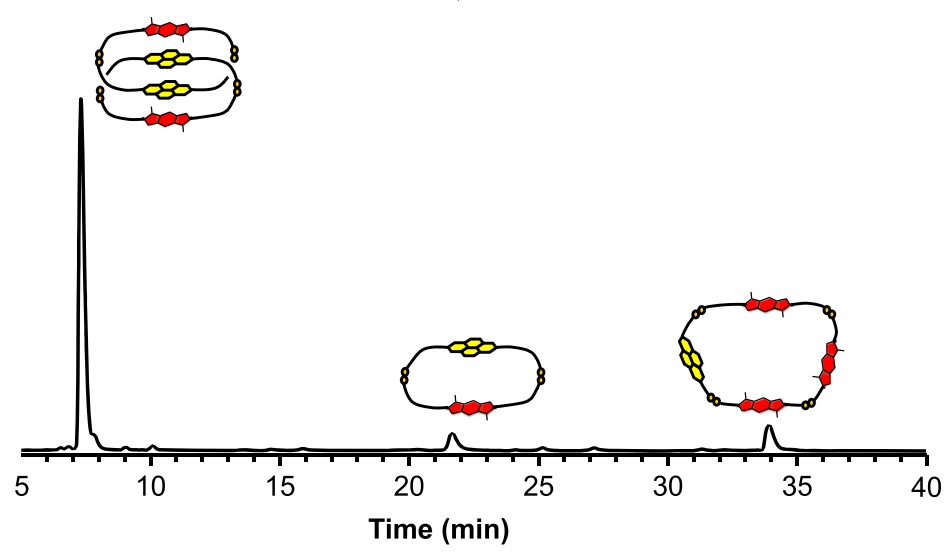

C

\begin{tabular}{|c|c|c|c|}
\hline Species & & \\
\hline i.d. & Cat I RRRR & $\mathbf{X}$ & $\begin{array}{c}\text { DDDA } \\
\text { (RRRR) } \\
\text { Macrocycle }\end{array}$ \\
\hline$\%$ in DCL & $75.83(0.25)$ & $16.38(0.05)$ & $7.79(0.26)$ \\
\hline
\end{tabular}

b

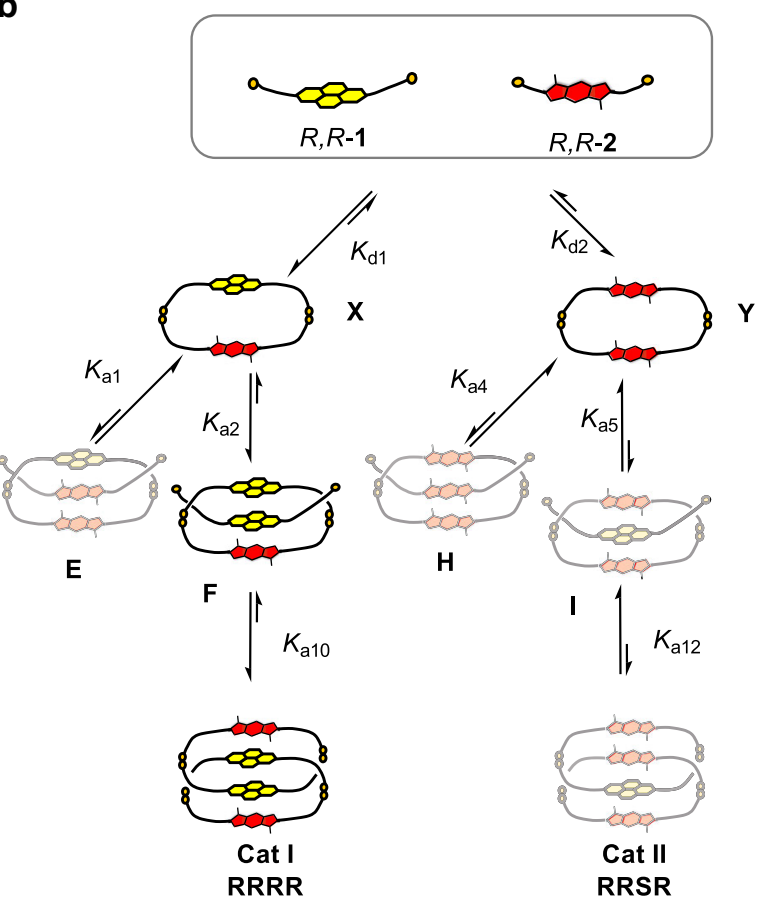

Fig. 2 Homochiral DCL. a Reverse-phase HPLC analysis at $389 \mathrm{~nm}$ of $R, R-\mathbf{1}: R, R-\mathbf{2}$ (1:1 molar ratio, $5 \mathrm{mM}$ total concentration) library in the presence of $1 \mathrm{M}$ $\mathrm{NaNO}_{3} ; \mathbf{b}$ schematic representation of equilibria showing the pathway for the formation of Cat I RRRR and $\mathbf{c}$ table containing the yields for each species in $\mathrm{DCL}$; the integrations were done in triplicate and the RMSD is reported in parenthesis along with the value.

In contrast to this, the kinetic analysis of the DCL of $S, S-\mathbf{1}$ and $R, R-2$ (Supplementary Figs. 67-68) reveals that the corresponding heterodimer $\mathbf{Z}$ (Fig. 3) forms in similar concentration as homodimer $\mathbf{Y}$ (a species not observed in the homochiral DCL, vide supra). Cat II RSRR is assembled through the complexation of an NDI core (S,S-1) in the cavity of $\mathbf{Y}$, followed by ring closure. This process outcompetes the threading of the NDI core in the heterodimer $\mathbf{Z}$, as indicated by the lack of the diastereomer of Cat I RSSR in this library.

The kinetic analysis of the DCL containing a racemic mixture of 1 and $R, R-2$ reveals a very complex behaviour, in which all building blocks combine to form linear and cyclic species. The fastest forming species are the linear homochiral heterodimer $\mathbf{X}$ (20\% of the library, based on HPLC peak integration after $30 \mathrm{~min}$ of equilibration), followed by its diastereomer, the heterochiral heterodimer $\mathbf{Z}(12 \%)$. This chiral differentiation is further amplified at the cyclisation stage, as after $85 \mathrm{~min}$ of DCL equilibration, the homochiral heterodimer $\mathbf{X}$ represents $13 \%$ of the DCL, while the heterochiral heterodimer $\mathbf{Z}$ amounts to only $4 \%$. At this stage, the homodimer $\mathrm{Y}$ accounts for $8 \%$ of the DCL (Supplementary Figs. 79-80). Based on these observations, we propose that the structural divergence observed is due to two factors: (a) the preferential formation of two of the possible nine pseudorotaxanes (Fig. 4), and (b) the relative stability of the final reaction products, Cat I RRRR and Cat II RSRR.

The first species to form in all libraries are the linear and cyclic homodimers and heterodimers ${ }^{25}$. The cyclic homochiral heterodimer $\mathbf{X}$ forms in a larger proportion than the corresponding heterochiral heterodimer $\mathbf{Z}$ in the racemic libraries, which implies that the chirality mismatch in $\mathbf{Z}$ leads to less effective aromatic stacking interactions between the NDI and BDT cores. This is further supported by the fact that in the $S, S-\mathbf{1}, R, R-\mathbf{2}$ library $\mathbf{Z}$ and the BDT homodimer $\mathbf{Y}$ are present in similar proportion despite that statistically, the ratio Z:Y should be 2:1 (the formation of Cat II RSRR does not influence the Z:Y ratio as it contains equal amount of both molecules in its composition).

The second key stage that contributes to the structural divergence is the formation of the pseudorotaxanes via the threading of a building block or linear dimer through the hetero or homodimers $\mathbf{X}, \mathbf{Y}$ or $\mathbf{Z}$. The homochiral heterodimer $\mathbf{X}$ will thread a NDI over a BDT core, accounting for better donor-acceptor stacking interactions $\left(K_{\mathrm{a} 2}, K_{a 3} » K_{\mathrm{a} 1}\right)$. Furthermore, the threading with a $R, R-\mathbf{1}$ is favoured over a $S, S-\mathbf{1}$ as indicated by the formation of Cat I RRRR via pseudorotaxane $\mathbf{F}$ over the corresponding diastereomer G. Control experiments, in which enantiopure serine analogues of $\mathbf{1}$ were used as competitors 
a
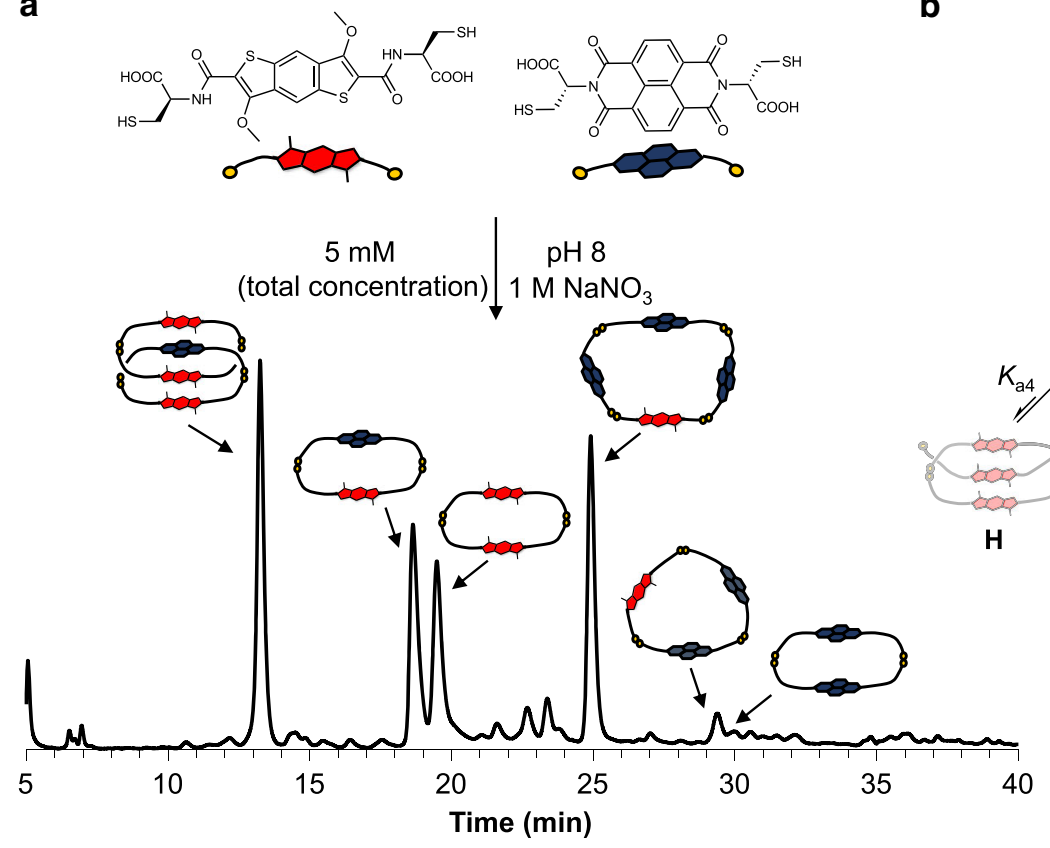

b

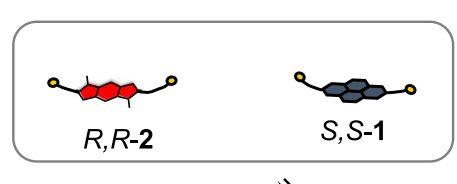

R,R

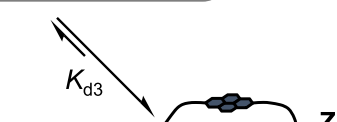

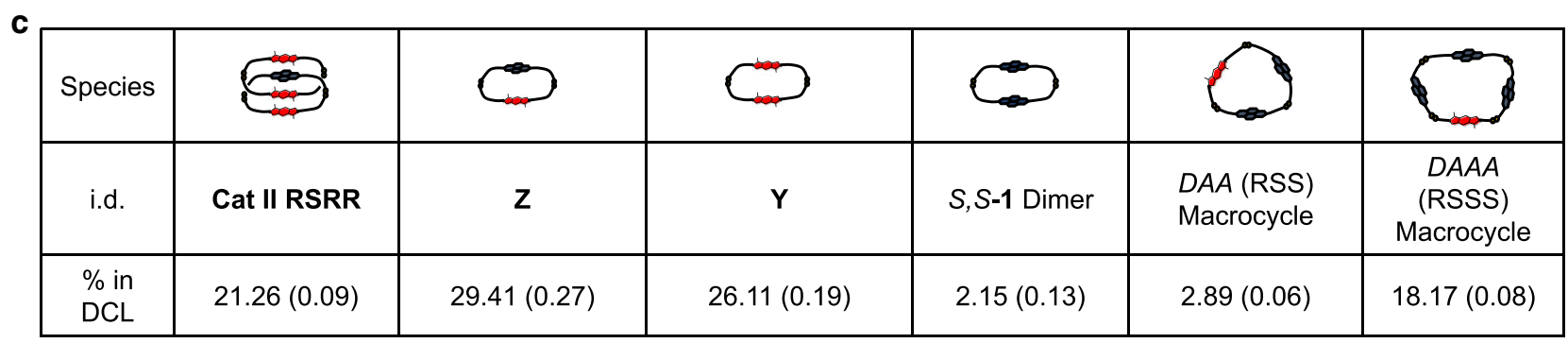

Fig. 3 Heterochiral DCL. a Reverse-phase HPLC analysis at $389 \mathrm{~nm}$ of S,S-1:R,R-2 (1:1 molar ratio, $5 \mathrm{mM}$ total concentration) library in the presence of $1 \mathrm{M}$ $\mathrm{NaNO}_{3} ; \mathbf{b}$ schematic representation of equilibria showing the pathway for the formation of Cat II RSRR and $\mathbf{c}$ table containing the yield for each species in $\mathrm{DCL}$; the integrations were done in triplicate and the RMSD is reported in parenthesis along with the value.

in DCLs containing $R, R-\mathbf{1}$ and $R, R-\mathbf{2}$, confirmed this hypothesis as the NDI-S,S-serine lowers the yield of Cat I RRRR in comparison to NDI- $R, R$-serine (Supplementary Fig. 47), thus $K_{\mathrm{a} 2}$ $>K_{\mathrm{a} 3}$ " $K_{\mathrm{a} 1}$ (NDI-S,S-serine is an analogue of $R, R-\mathbf{1}$, as $S$-serine and $R$-cysteine are both L-amino acids). We used DCLSim ${ }^{26}$ to simulate the DCLs in the presence and absence of the NDI-serine competitors (DCLSim part in Supporting Information). The simulated DCLs distributions are in good agreement with the observed ones and confirmed that $K_{\mathrm{a} 2}>K_{\mathrm{a} 3}$.

The BDT homodimer $(\mathbf{Y})$ is the dominant species after $130 \mathrm{~min}$ in the DCL of $S, S-1, R, R-2$ and, based on the stacking interactions, it likely generates the most stable pseudorotaxane (J) with $S, S-1$ (Supplementary Figs. 67-68). In the racemic DCL, the competing species I does not form to a large extent, as confirmed by control DCLs with the enantiopure NDI-serine analogues and by the lack of the corresponding catenane (Supplementary Fig. 74). Additionally, UV-Vis titrations of $\mathbf{Y}$ with the DCC inert serine analogues ${ }^{27}$ highlighted a higher association constant between $\mathbf{Y}$ and NDI- $R, R$-serine compared to NDI-S,S-serine (Supplementary Figs. 86-87), therefore $K_{\mathrm{a} 6}>K_{\mathrm{a} 5}$. This inequality is supported by DCLSim calculations, which also indicate that heterodimer $\mathbf{Z}$ binds $S, S-1$ more strongly than $R, R-\mathbf{1}$, with $K_{\mathrm{a} 9}>K_{\mathrm{a} 8}$.

Pseudorotaxanes $\mathbf{E}, \mathbf{K}$, and $\mathbf{H}$ have unfavourable donor-donor stacking interactions ${ }^{28,29}$ due to the formation of an $A D D$ stack $(\mathbf{E}, \mathbf{K})$ and a $D D D$ stack $(\mathbf{H})$ which allows us to conclude that relationship of the association constant is $K_{\mathrm{a} 1}, K_{\mathrm{a} 7}>K_{\mathrm{a} 4}$.
As in all DCLs, the stability of the final products plays a crucial role in the composition at equilibrium. Cat I RRRR and Cat II RSRR are more stable than their corresponding diastereomers as indicated by molecular modelling (PM7, COSMO water model, see "Methods" section). The Cat I RRRR is about $36.5 \mathrm{~kJ} / \mathrm{mol}$ more stable than Cat I RSSR, and about $50 \mathrm{~kJ} / \mathrm{mol}$ more stable than Cat I RSRR. There is a difference in stability for the Cat II diastereomers, where the Cat II RSRR is more stable than Cat II RRRR by almost $9 \mathrm{~kJ} / \mathrm{mol}$. In this case, the selectivity for Cat II RSRR in the racemic DCL is likely due to the incorporation of $R, R-\mathbf{1}$ in Cat I RRRR at the expense of Cat II RRRR. These differences are not surprising as the stacked structure of the catenanes forces the carboxylate groups to be in close proximity, therefore a change in the point chirality imposes new steric and electrostatic repulsion demands on the overall structure (Supplementary Table 6).

\section{Discussion}

In conclusion, we have merged dynamic combinatorial chemistry with structurally divergent reactions on racemic mixtures. The structural divergence is due to the formation of two diastereomeric macrocycles and stereospecific formation of pseudorotaxanes under disulphide exchange conditions. This is an example of aqueous systems chemistry in which small changes in the building blocks' structures lead to complex molecules through 


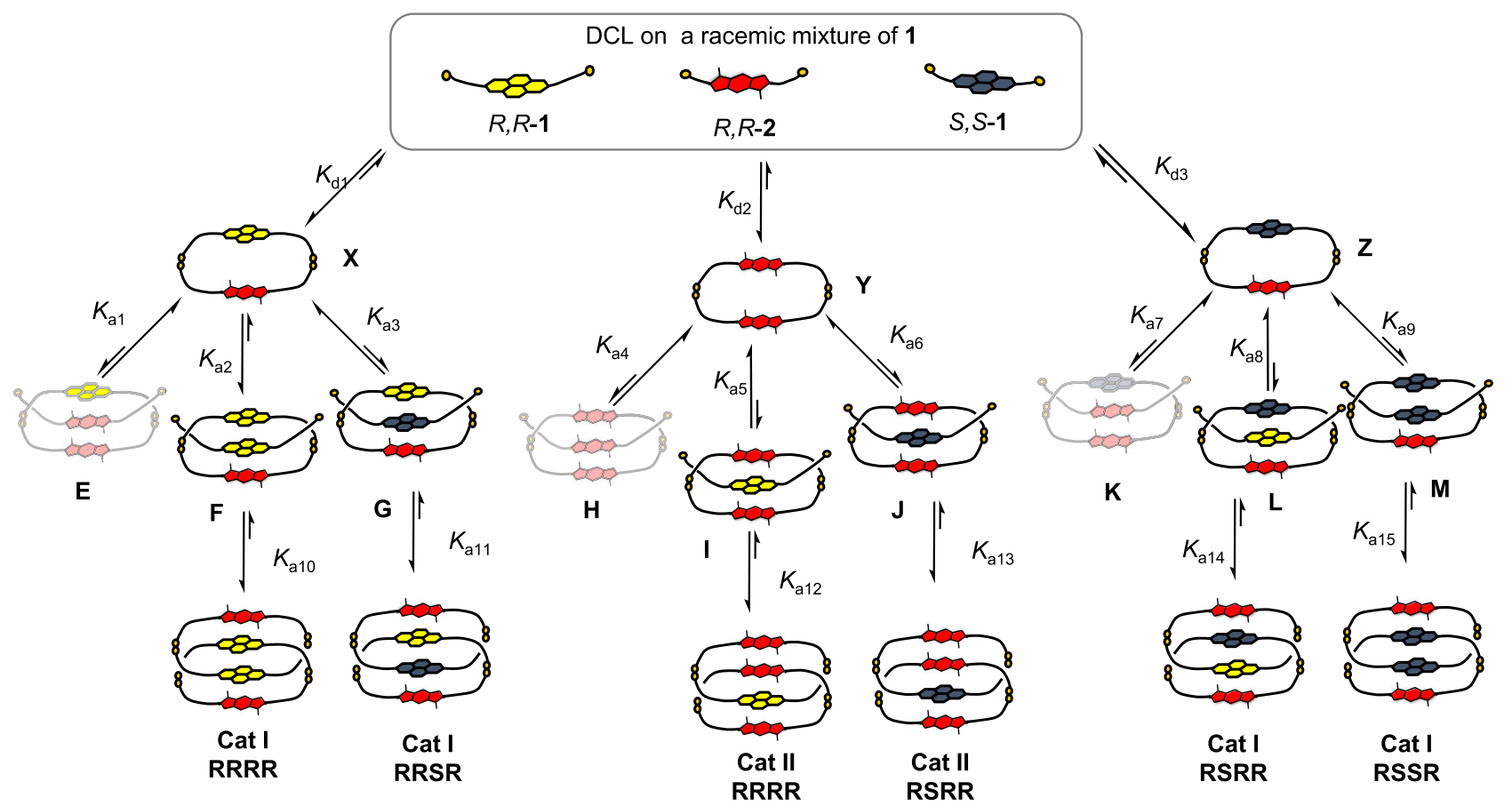

Fig. 4 Rationalising the DCLs outcome. Schematic representation (simplified) of the pathways leading to Cat I RRRR and Cat II RSRR. The equilibrium arrows show the relative position of the equilibrium based on theoretical and experimental data. The faded structures are either present in less than $1 \%$ or not present in the DCLs analysed. $K_{d}=$ dimerisation constant, $K_{\mathrm{a}}=$ association constant.

interconnected mechanisms. This SDRRM is driven by supramolecular interactions and highlights the importance of stereospecific supramolecular recognition observed in Nature. Here, we showed how a chiral reagent reacts with a racemic mixture to lead to compounds with non-isomeric structures based on which enantiomer is incorporated in the final product. This type of structural divergence based on supramolecular interactions could be the seed that led to the demise of one set of protobiomolecules resulting in the observed homochirality of the biological world. We are currently investigating the formation of other conformationally restricted molecules from racemic mixtures.

\section{Methods}

Materials and characterisation. All reagents were purchased from commercial suppliers: Acros Organics, Alfa Aesar, Sigma Aldrich, TCI Europe, Fluorochem, and used without further purification. ${ }^{1} \mathrm{H}$ and ${ }^{13} \mathrm{C}$ nuclear magnetic resonance (NMR) spectra were recorded on $500 \mathrm{MHz}$ Agilent Propulse or $500 \mathrm{MHz}$ Bruker Avance II $+\left({ }^{1} \mathrm{H} 500 \mathrm{MHz},{ }^{13} \mathrm{C} 125 \mathrm{MHz}\right)$ instruments, as stated. Chemical shifts $(\delta)$ are reported in parts per million (ppm). Coupling constants are reported in Hertz (Hz), and signal multiplicity is denoted as singlet (s), doublet (d), doublet of doublet (dd), triplet (t), dt (doublet of triplets), td (triplet of doublets), h (heptet), multiplet (m), and broad (br). All spectra were acquired at $25^{\circ} \mathrm{C}$ and were referenced to the residual solvent peaks. The common solvent impurities in ${ }^{1} \mathrm{H}$ and ${ }^{13} \mathrm{C}$ NMR in small amounts were water, acetone or DMF. COSY, NOESY, and HSQC spectra were recorded on a Bruker AV III $500 \mathrm{MHz}$ fitted with Prodigy (nitrogencooled) cryoprobe. The microwave reactions were carried out in either CEM Discover or CEM Explorer 12. LC-MS studies were carried out on a Thermo Surveyor PDA Plus LC and LCQ classic ESI MS. Data was processed using the XCalibur software. The individual HPLC/LC-MS methods are detailed in the LCMS Analysis Section. The LC CD data was acquired on a Jasco CD 2095 connected to a SS420 $\times$ A/D converter. All the CD data was acquired on an Applied Photophysics Chirascan spectro-photometer equipped with a Peltier temperature controller.

DCL set-up. A $5 \mathrm{mM}$ library was prepared by dissolving the building block in $10 \mathrm{mM}$ aqueous $\mathrm{NaOH}$, followed by titration with $100 \mathrm{mM} \mathrm{NaOH} / 100 \mathrm{mM} \mathrm{HCl}$ to adjust the $\mathrm{pH}$ to 8 . The DCL solutions were stirred in close-capped vials and analysed by LC-MS. Preparative libraries ( $\geq 5 \mathrm{~mL}$ scale) were made using the same method as for the analytical libraries.
LC-MS method. ESI-MS spectra (negative ion) were acquired with drying temperature of $250^{\circ} \mathrm{C}$, spray current $0.5 \mu \mathrm{A}$, sheath gas flow of $40 \mathrm{arb}$, spray voltage was set to $4.5 \mathrm{kV}$, capillary voltage $13 \mathrm{~V}$, tube lens $-15.0 \mathrm{~V}$. The mass range was set from $\mathrm{m} / \mathrm{z}$ 150-2000, the number of microscans in scan time was 5 and the maximum injection time was $150.0 \mathrm{~ms}$.

MS/MS setting. Parent mass $(\mathrm{m} / \mathrm{z})$ : dependant on the species, ionisation width $(\mathrm{m} / \mathrm{z})$ : 4.0 , normalised collision energy $(\%): 20$, activation Q: 0.250 , activation time (ms) 30.0, the number of microscans in scan time was 5 and the maximum injection time was $150.0 \mathrm{~ms}$.

Analytical HPLC method for DCLs of Cat I RRRR (5 mM). Column: Phenomenex Develosil RPAqueous- $3 \mathrm{C}_{30}, 5 \times 0.3 \mathrm{~cm}, 3 \mu \mathrm{m}$. Injection volume: $2 \mu \mathrm{L}$; flow rate: $1 \mathrm{~mL} / \mathrm{min}$; temperature: $39^{\circ} \mathrm{C}$; run time: $7 \mathrm{~min}$; elution profile: See Supplementary Table 1 .

Preparative HPLC method for DCLs of Cat I RRRR (5 mM). Column: Phenomenex Develosil Combi-RP C $30,5 \times 2 \mathrm{~cm}, 5 \mu \mathrm{m}$; injection volume: $900 \mu \mathrm{L}$; Flow rate: $6 \mathrm{~mL} / \mathrm{min}$; temperature: $39^{\circ} \mathrm{C}$; Run time: $34 \mathrm{~min}$; elution profile: see Supplementary Table 2 .

Analytical HPLC method for DCLs of Cat II RSRR (5 mM). Column: Phenomenex Develosil RPAqueous- $3 \mathrm{C}_{30}, 5 \times 0.3 \mathrm{~cm}, 3 \mu \mathrm{m}$. Injection volume: $2 \mu \mathrm{L}$; flow rate: $1 \mathrm{~mL} / \mathrm{min}$; temperature: $39^{\circ} \mathrm{C}$; run time: $7 \mathrm{~min}$; elution profile: see Supplementary Table 3 .

Preparative HPLC method for DCLs of Cat II RSRR (5 mM). Column: Phenomenex Develosil Combi-RP $\mathrm{C}_{30}, 5 \times 2 \mathrm{~cm}, 5 \mu \mathrm{m}$; injection volume: $600 \mu \mathrm{L}$; flow rate: $6 \mathrm{~mL} / \mathrm{min}$; temperature: $39^{\circ} \mathrm{C}$; run time: $24 \mathrm{~min}$; elution profile: See Supplementary Table 4 .

Analytical HPLC method for DCLs of all libraries ( $5 \mathrm{mM}$ ). Column: Restek Ultra BiPhenyl, $5 \times 0.3 \mathrm{~cm}, 3 \mu \mathrm{m}$. Injection volume: $3 \mu \mathrm{L}$; flow rate: $0.5 \mathrm{~mL} / \mathrm{min}$; temperature: $39^{\circ} \mathrm{C}$; run time: $43 \mathrm{~min}$; elution profile: See Supplementary Table 5 .

Circular dichroism settings for isolated Cat I RRRR and Cat II RSRR analysis The isolated catenane (after purity checking by HPLC), Cat I RRRR or Cat II RSRR, was assessed for optical and chiroptical properties. The catenane has a CD band across the $550 \mathrm{~nm}$ region, which is expected for $D-A$ interaction. The experiments were done in a 1 or $10 \mathrm{~mm}$ pathlength cuvettes depending on the concentration, with a $4 \mathrm{~nm}$ bandwidth. Scan mode: 1 point $/ \mathrm{nm}$, time-per-poin 
$0.5 \mathrm{~s}$. The VT experiments $\left(10^{\circ} \mathrm{C}\right.$ increment and setting time $\left.300 \mathrm{~s}\right)$ were performed in the same cuvette using a stirrer bar.

UV-Vis titration of NDI- $\boldsymbol{R}, \boldsymbol{R} / \mathbf{S}, \mathbf{S}$-serine into $\mathbf{Y}$. Y was prepared by dissolving the $R, R-2$ building block in water at $\mathrm{pH} 8$ and allowed to equilibrate over two days, yielding $\mathbf{Y}$ homodimer as the major product (more than $90 \%$ ). From this stock solution, two solutions $(3.57 \mathrm{mM})$ were prepared: one containing the homodimer and $1 \mathrm{M} \mathrm{NaNO}_{3}$ (soln. T) and the other with NDI- $R, R$-serine or NDI- $S$, S-serine (35.7 mM, 10 equiv) and $1 \mathrm{M} \mathrm{NaNO}_{3}$ (soln. U). Small aliquots of soln. U were gradually added into soln. $\mathrm{T}$, having $0.1,0.3,0.5,0.7,0.8,0.9,1.0,1.1,1.2,1.5,2.0$, 3.0, 4.0, and 5.0 equiv of NDI-serine at each titration point in the final solution.

Testing reversibility with DTT. In order to test the reversible behaviour of the reactions, both Cat I RRRR and Cat II RSRR were subjected to dithiothreitol (DTT) after isolation. DTT is a dithiol-based compound that can restart the disulphide exchange and reform the library.

Computational studies. Geometry optimisation was performed using Avogadro ${ }^{30}$ (Force field: UFF, Algorithm: Conjugate gradients). This was followed by MOPAC $2016^{31}$ (Version 18.117 M) optimisation PM7 semi-empirical optimisation using the COSMO water model with a $0.1 \mathrm{kcal} / \mathrm{mol} /$ Ångstrom convergence factor, using Gabedit $^{32} 2.5 .0$ as interface.

\section{Data availability}

The data that support the findings of this study are available within the paper and its Supplementary Information with https://doi.org/10.1038/s41467-020-17321-2 All data underlying the findings of this work are available from the corresponding author upon reasonable request.

Received: 15 October 2018; Accepted: 16 June 2020;

Published online: 15 July 2020

\section{References}

1. Miller, L. C. \& Sarpong, R. Divergent reactions on racemic mixtures. Chem. Soc. Rev. 40, 4550-4562 (2011)

2. Nadeau, E., Ventura, D. L., Brekan, J. A. \& Davies, H. M. L. Controlling factors for $\mathrm{C}-\mathrm{H}$ functionalization versus cyclopropanation of dihydronaphthalenes. J. Org. Chem. 75, 1927-1939 (2010).

3. Tanaka, K., Hagiwara, Y. \& Hirano, M. Rh-catalyzed [4+2] annulation of 4alkynals with isocyanates and its application to the parallel kinetic resolution of unfunctionalized 4-alkynals. Angew. Chem. Int. Ed. 45, 2734-2737 (2006).

4. Kato, K., Motodate, S., Takaishi, S., Kusakabe, T. \& Akita, H. Parallel kinetic resolution of propargyl ketols: formal synthesis of (+)-bakkenolide A. Tetrahedron 64, 4627-4636 (2008).

5. Tanaka, K. \& Fu, G. C. Parallel kinetic resolution of 4-alkynals catalyzed by Rh (I)/Tol-BINAP: synthesis of enantioenriched cyclobutanones and cyclopentenones. J. Am. Chem. Soc. 125, 8078-8079 (2003).

6. Davies, H. M. L. \& Walji, A. M. Direct synthesis of $(+)$-erogorgiaene through a kinetic enantiodifferentiating step. Angew. Chem. Int. Ed. 44, 1733-1735 (2005).

7. Davies, H. M. L., Dai, X. \& Long, M. S. Combined C-H activation/cope rearrangement as a strategic reaction in organic synthesis: total synthesis of (-)-colombiasin A and (-)-elisapterosin B. J. Am. Chem. Soc. 128, 2485-2490 (2006).

8. Gansäuer, A., Shi, L., Otte, M. Catalytic Enantioselective Radical Cyclization via Regiodivergent Epoxide Opening. J. Am. Chem. Soc. 132, 11858-11859 (2010).

9. Cougnon, F. B. L., Ponnuswamy, N., Jenkins, N. A., Pantoş, G. D. \& Sanders, J. K. M. Structural parameters governing the dynamic combinatorial synthesis of catenanes in water. J. Am. Chem. Soc. 134, 19129-19135 (2012).

10. Dehkordi, M. E., Luxami, V. \& Pantoş, G. D. High-yielding synthesis of chiral donor-acceptor catenanes. J. Org. Chem. 83, 11654-11660 (2018).

11. Soai, K., Shibata, T., Morioka, H. \& Choji, K. Asymmetric autocatalysis and amplification of enantiomeric excess of a chiral molecule. Nature 378, 767 (1995).

12. Guijarro, A. \& Yus, M. The Origin of Chirality in the Molecules of Life: A Revision from Awareness to the Current Theories and Perspectives of this Unsolved Problem. (Royal Society of Chemistry, 2008).

13. Brown, J. M. et al. Amplification of Chirality. Vol. 284 (Springer, Berlin Heidelberg, 2008).

14. Pengo, P., Pantoş, G. D., Otto, S. \& Sanders, J. K. M. Efficient and mild microwave-assisted stepwise functionalization of naphthalenediimide with aamino acids. J. Org. Chem. 71, 7063-7066 (2006)
15. Au-Yeung, H. Y., Pantoş, G. D. \& Sanders, J. K. M. Amplifying different [2] catenanes in an aqueous donor-acceptor dynamic combinatorial library. J. Am. Chem. Soc. 131, 16030-16032 (2009).

16. Au-Yeung, H. Y., Pantoş, G. D. \& Sanders, J. K. Dynamic combinatorial synthesis of a catenane based on donor-acceptor interactions in water. Proc. Natl Acad. Sci. 106, 10466-10470 (2009).

17. Au-Yeung, H. Y., Pantos, G. D. \& Sanders, J. K. M. A water soluble donor-acceptor [2] catenane that can switch between a coplanar and a geminisign conformation. Angew. Chem. Int. Ed. 49, 5331-5334 (2010)

18. Ponnuswamy, N., Cougnon, F. B. L., Clough, J. M., Pantoş, G. D. \& Sanders, J K. M. Discovery of an organic trefoil knot. Science 338, 783-785 (2012).

19. Ponnuswamy, N., Cougnon, F. B. L., Pantos, G. D. \& Sanders, J. K. M. Homochiral and meso figure eight knots and a solomon link. J. Am. Chem. Soc. 136, 8243-8251 (2014).

20. Ota, S. et al. Synthesis and properties of a benzo[1,2-b:4,5-b']dithiophene core $\pi$-system that bears alkyl, alkylthio and alkoxy groups at 3,7-positions. RSC Adv. 3, 12356-12365 (2013).

21. Pai, R. K., Ahipa, T. N. \& Hemavathi, B. Rational design of benzodithiophene based conjugated polymers for better solar cell performance. RSC Adv. 6, 23760-23774 (2016).

22. Fujita, M., Ibukuro, F., Ogura, K. \& Hagihara, H. Quantitative self-assembly of a [2] catenane from two preformed molecular rings. Nature 367, 720-723 (1994).

23. Since the disulfide reductions do not occur in the experimental conditions, these equilibria do not describe the actual processes happening in the DCLs. However the relative difference of the Ka of various library members provides a stability ranking.

24. Li, J., Nowak, P., Fanlo-Virgós, H. \& Otto, S. Catenanes from catenanes: quantitative assessment of cooperativity in dynamic combinatorial catenation. Chem. Sci. 5, 4968-4974 (2014).

25. The $R, R-\mathbf{1}$ and $S, S-\mathbf{1}$ homo and heterochiral homodimers are too tight to allow threading of an aromatic core through their cavity, thus remaining inactive supramolecular interactions in the DCL. These species could be 'reservoirs' of NDI building blocks throughout the equilibration.

26. Corbett, P. T., Otto, S. \& Sanders, J. K. M. Correlation between host-guest binding and host amplification in simulated dynamic combinatorial libraries. Chemistry 10, 3139-3143 (2004).

27. Titrations performed with $R, R-\mathbf{1}$ and $S, S-\mathbf{1}$ reinitialize the disulphide exchange, see Supplementary Figs. 58-59, 69-70.

28. Cubberley, M. S. \& Iverson, B. L. H NMR investigation of solvent effects in aromatic stacking interactions. J. Am. Chem. Soc. 123, 7560-7563 (2001).

29. Prentice, G. M., Pascu, S. I., Filip, S. V., West, K. R. \& Pantoş, G. D. Aromatic donor-acceptor interactions in non-polar environments. Chem. Commun. 51, 8265-8268 (2015)

30. Hanwell, M. D. et al. Avogadro: an advanced semantic chemical editor, visualization, and analysis platform. J. Cheminform. 4, 17 (2012).

31. Stewart, J. J. P. Optimization of parameters for semiempirical methods VI more modifications to the NDDO approximations and re-optimization of parameters. J. Mol. Model. 19, 1-32 (2013).

32. Allouche, A.-R. Gabedit-A graphical user interface for computational chemistry softwares. J. Comput. Chem. 32, 174-182 (2011).

\section{Acknowledgements}

We thank the Engineering and Physical Sciences Research Council (EPSRC, EB-CH1264) for funding and the EPSRC National Mass Spectrometry Service Centre (Swansea, U.K.) for high resolution mass spectrometry. We thank P. J. Pantoș, D. M. Răsădean, M. T. Huggins for helpful discussions.

\section{Author contributions}

T.M.G. carried out the experimental work. T.M.G. and G.D.P. did the computational work. G.D.P. and T.M.G. designed the experiments. Both authors contributed to the analysis of the results and the writing of the manuscript. G.D.P. directed the research.

\section{Competing interests}

The authors declare no competing interests.

\section{Additional information}

Supplementary information is available for this paper at https://doi.org/10.1038/s41467020-17321-2.

Correspondence and requests for materials should be addressed to G.D.P.

Peer review information Nature Communications thanks the anonymous reviewer(s) for their contribution to the peer review of this work.

Reprints and permission information is available at http://www.nature.com/reprints

Publisher's note Springer Nature remains neutral with regard to jurisdictional claims in published maps and institutional affiliations. 
(c) (i) Open Access This article is licensed under a Creative Commons Attribution 4.0 International License, which permits use, sharing, adaptation, distribution and reproduction in any medium or format, as long as you give appropriate credit to the original author(s) and the source, provide a link to the Creative Commons license, and indicate if changes were made. The images or other third party material in this article are included in the article's Creative Commons license, unless indicated otherwise in a credit line to the material. If material is not included in the article's Creative Commons license and your intended use is not permitted by statutory regulation or exceeds the permitted use, you will need to obtain permission directly from the copyright holder. To view a copy of this license, visit http://creativecommons.org/ licenses/by/4.0/.

(C) The Author(s) 2020 